\title{
Use of Antifungals and Outcomes Among Inpatients at Risk of Invasive Aspergillosis or Mucormycosis in the USA: A Retrospective Cohort Study
}

\author{
Katherine Stull · Elizabeth Esterberg · Mayank Ajmera • \\ Sean Candrilli · Therese M. Kitt · James R. Spalding • Vanessa Perez Patel
}

Received: August 22, 2019 / Published online: October 9, 2019

(C) The Author(s) 2019

\begin{abstract}
Introduction: Prophylaxis and treatment of invasive aspergillosis (IA) and mucormycosis (IM) within a real-world US inpatient setting is undocumented since the introduction of isavuconazole. This retrospective medical record review aimed to describe characteristics, triazole use, and outcomes among inpatients across the USA who initiated antifungal monotherapy (AFMT) as prophylaxis or treatment of IA/IM.

Methods: A convenience sample of US physicians abstracted data from randomly selected records of hospitalized patients aged $\geq 18$ years initiating AFMT (amphotericin B, isavuconazole, voriconazole, or posaconazole) as prophylaxis or treatment of IA/IM between 2013 and 2017. Retrieved data included background characteristics, dosage and duration of AFMT, healthcare resource use, and survival. Characteristics and outcomes were compared
\end{abstract}

Enhanced digital features To view enhanced digital features for this article go to https://doi.org/10.6084/ m9.figshare.9861131.

K. Stull $(\varangle) \cdot$ E. Esterberg · M. Ajmera · S. Candrilli Health Economics, RTI Health Solutions, Research Triangle Park, NC, USA

e-mail: kstull@rti.org

T. M. Kitt · J. R. Spalding · V. P. Patel Medical Affairs, Astellas Pharma Global Development, Inc., Northbrook, IL, USA (prophylaxis vs treatment) using Fisher's exact and one-way analysis of variance tests where applicable. Exploratory Kaplan-Meier analyses described overall and inpatient survival.

Results: Physicians $(n=23)$ retrieved 124 patient records (43 prophylaxis; 81 treatment). Median duration of first-line AFMT was 14 days (range 1-603 days) and 19 days (range 3-351 days) in the prophylaxis and treatment groups, respectively. One patient received second-line therapy. Median duration of hospitalization was 29 days (range 4-259 days) and 31 days (range 6-980 days) in the prophylaxis and treatment groups, respectively. Admission to intensive care occurred in $14 \%$ and $52 \%$ of patients in the prophylaxis and treatment groups, respectively. At the time of data retrieval, overall and inpatient survival rates in the prophylaxis group were $88 \%$ and $87 \%$, respectively, and in the treatment group were $66 \%$ and $76 \%$, respectively.

Conclusions: This study documented realworld prophylactic and therapeutic AFMT use for IA/IM and associated outcomes among hospitalized patients in the USA since approval of isavuconazole. IA/IM were associated with lengthy hospital stays commonly requiring intensive care. Prophylactic and therapeutic AFMT dosages and duration generally followed recommendations and switching between agents was rare.

Funding: Astellas Pharma Global Development, Inc., Northbrook, IL, USA. 
Keywords: Antifungals; Fungal infection; Inpatient; Invasive aspergillosis; Invasive mucormycosis; Mold-active triazoles; Retrospective

\section{INTRODUCTION}

Invasive fungal infections (IFIs) such as invasive aspergillosis (IA) and mucormycosis (IM) can result in severe disease and are potentially fatal [1-5]. An increase in the number of deaths from IFIs has been documented [6-9], and rates of IAand IM-related hospitalizations have increased since 2000 [10]. As the number of patients treated with immunosuppressive therapy and intensive chemotherapy regimens grows, the incidence of these infections has increased [2].

Despite treatment advancements and clinical guidelines, the prevention, diagnosis, and treatment of IFIs remain challenging. Randomized clinical trials have investigated the efficacy of mold-active triazoles [11-13], but are limited in the depth of information they can provide due to the rarity of the conditions, the challenges of recruiting severely ill and immunocompromised patients, and the heterogeneity of the underlying disease. Observational cohort studies provide opportunities to examine realworld current approaches to the diagnosis and management of IFIs, and offer a greater ability to examine the breadth of both the underlying heterogeneity and the severity of the disease [14-17]. The results of such studies have broadened our understanding of unmet treatment needs and have informed the development of current guidelines [18]. However, no real-world evidence of prevention and treatment strategies within this patient population has been published since the introduction of isavuconazole in the USA in 2015.

The study presented herein examines realworld prevention and treatment strategies for IA/IM in the inpatient setting in the USA since 2013. Specifically, this study aimed to describe the characteristics of hospitalized patients across the USA who initiated prophylaxis or treatment with mold-active triazoles or amphotericin B for IA or IM; document dosage and duration of mold-active triazole use; and evaluate patient outcomes pertinent to healthcare resource use and survival.

\section{METHODS}

\section{Study Design and Population}

A retrospective cohort study of patient medical records from hospitals across the USA was conducted. Records were eligible if patients were aged 18 years or older at the time of hospitalization and had initiated antifungal monotherapy (AFMT) during the hospitalization for either prophylaxis or treatment of IA or IM between January 1, 2013 and August 31, 2017. The antifungal agents evaluated were prespecified and based on current clinical guidelines [18]: amphotericin B (any formulation), isavuconazole, voriconazole, and posaconazole. No restrictions were placed on the use of antifungals following initiation of these agents. Eligible physicians were hospital-based and currently managing patients with IFIs, had treated at least one patient for IA/IM per year, had acted as a key decision-maker in the management of IA/IM, and were experienced with the antifungal agents of interest.

\section{Data Retrieval Methods}

Data were retrieved between January 26, 2018 and March 9, 2018, inclusive. Data from hospitalization until the last medical record entry or documented date of death were entered by physicians into an anonymized, web-based electronic data retrieval form developed by the study authors (KS, VPP). During development, the data retrieval form was reviewed and tested by two eligible physicians within the USA to assess its functionality and availability of the requested data elements.

A convenience sampling approach was used to recruit physicians, and no quotas were applied [19]. A quasi-random method was applied for patient selection by asking physicians to select four records for patients whose last name began with a randomly generated letter (A through $\mathrm{Z}$, inclusive). If no eligible 
patient record was identified, the physician was asked to select a patient whose last name began with the next letter in alphabetical order. Physicians continued this process until 4 patients per institution had been identified (one for each of amphotericin B, isavuconazole, voriconazole, and posaconazole).

The data retrieved consisted of: sociodemographic/clinical characteristics at the time of hospitalization; dates of hospitalization and antifungal therapy initiation; antifungal drugs used, including reason for initiation; therapy start/stop dates, dose, and frequency; changes in therapy regimens and attributed reasons for change, if any; hospital discharge date; admission to an intensive care unit (ICU); need for mechanical ventilation; hospital readmission within 30 days of discharge; vital status at the time of data abstraction (i.e., dead or alive); date of death; and date of last medical record entry.

All procedures performed in studies involving human participants were in accordance with the ethical standards of RTI International's institutional review board (Research Triangle Park, North Carolina) and with the 1964 Helsinki declaration and its later amendments or comparable ethical standards. Informed consent was not sought due to the nature of the study: all retrieved data were anonymous and were those collected as part of routine diagnosis and treatment. There was no effect of the review on patient care. Physicians abstracting the data were treating physicians who had legitimate access to the medical records. RTI International's institutional review board determined that this study met all criteria for exemption from ethical considerations.

\section{Statistical Analysis}

Patient characteristics are described by reason for initiation of AFMT (i.e., prophylaxis or treatment). Outcomes data are described by reason for initiation of AFMT and by antifungal agent used.

Patient characteristics are reported as frequencies and percentages for categorical variables based on the number of patients with no missing data for each variable. Means, standard deviations (SDs), medians, and ranges are reported for continuous variables.

The retrieved data were used to calculate duration of hospitalization, duration of antifungal therapy, time to admission to ICU, time to hospital readmission within 30 days of discharge, rate of overall survival, and rate of inpatient survival. The date of the last medical record entry was used in calculations if patients were still receiving the AFMT of interest at the time of data retrieval. If patients died during the hospitalization or after discharge, the date of death was used to calculate durations. For overall survival, patients still alive at the time of data retrieval were censored at the date of the last available medical record entry. For inpatient survival, patients still alive and hospitalized at the time of data retrieval were censored at the date of the last available medical record entry, and discharged patients were censored at the date of discharge from hospital. Patients with undocumented status were excluded from the calculation of outpatient survival rates, and patients with an undocumented hospital discharge status were excluded from the calculation of inpatient survival rates.

Patient characteristics and outcomes were compared using Fisher's exact and one-way analysis of variance tests where applicable; $P$ values are reported, with $P \leq 0.05$ suggestive of statistically significant differences between patients initiating AFMT as prophylaxis or treatment. Exploratory Kaplan-Meier analyses were performed to describe overall and inpatient survival.

\section{RESULTS}

\section{Physician Characteristics}

Twenty-three physicians from 23 sites participated and abstracted data from 124 patient medical records $(n=31$ for each antifungal agent). Eight physicians abstracted data for 2 patients per antifungal agent (i.e., 8 records); 15 physicians abstracted data for one patient per antifungal agent (i.e., 4 records). Sixteen physicians abstracted data related to prophylaxis; 21 physicians abstracted data related to 
treatment. Geographically, 25\% of the physicians who abstracted data related to prophylaxis operated in the Midwest, 38\% in the Northeast, $19 \%$ in the West, and 19\% in the South of the USA. Of the physicians who abstracted data related to treatment, $29 \%$ operated in the Midwest, $24 \%$ in the Northeast, $29 \%$ in the West, and $19 \%$ in the South. Most physicians reported practicing in community (prophylaxis: $38 \%$; treatment: 52\%), academic/teaching (prophylaxis: 25\%; treatment: $24 \%$ ), or public (prophylaxis: $25 \%$; treatment: $19 \%$ ) hospitals. The most common medical specialties were infectious diseases (prophylaxis: 25\%; treatment: $38 \%$ ), oncology (prophylaxis: 38\%; treatment: 29\%), and intensive care (prophylaxis: 19\%; treatment: $19 \%)$.

\section{Patient Characteristics at Hospital Admission}

A total of 43 records were abstracted related to patients receiving AFMT as prophylaxis; a total of 81 related to patients receiving AFMT as treatment. Table 1 documents the demographic and background clinical characteristics of the sample, by reason for AFMT initiation (i.e., prophylaxis or treatment).

There were no significant differences in demographic characteristics between patients who initiated AFMT as prophylaxis versus treatment (Table 1$)$. Mean age $( \pm$ SD) was 53.4 $( \pm 14.6)$ and $51.3( \pm 16.0)$ years for patients receiving prophylaxis and treatment, respectively. Most patients were male (prophylaxis: 63\%; treatment: 64\%), overweight (mean body mass index, $\mathrm{kg} / \mathrm{m}^{2}[ \pm \mathrm{SD}]$ : prophylaxis: 26.6 [ \pm 3.3$]$; treatment: $25.9[ \pm 3.6])$, and white (prophylaxis: 65\%; treatment: 58\%).

IFIs occurred most commonly in the lung, blood, or sinuses; patients initiating AFMT as treatment were significantly more likely than those initiating AFMT as prophylaxis to have an IFI manifesting in the lung $(63 \%$ vs $35 \%$; $P<0.01)$ or blood $(42 \%$ vs $16 \% ; P<0.01)$ (Table 1). No significant differences in underlying diseases or host risk factors were observed based on the reason for AFMT initiation. The most frequent underlying diseases were acute myeloid leukemia and hematopoietic stem cell transplantation. Host risk factors most commonly noted in both the prophylaxis and treatment groups were prolonged neutropenia and fungal or bacterial infection. Less than 20\% of the sample had received antifungal therapy at any time prior to their hospitalization.

Patients receiving AFMT as prophylaxis were significantly more likely to be receiving posaconazole than those receiving AFMT as treatment (37\% vs $19 \% ; P<0.05$ ) (Table 1$)$. No significant differences were observed between the prophylaxis and treatment groups with respect to receipt of amphotericin B, isavuconazole, or voriconazole.

\section{Antifungal Therapy}

\section{Reason for AFMT Initiation}

Among patients initiating AFMT as treatment, most had proven infections (57\%) as defined by the European Organization for Research and Treatment of Cancer/Invasive Fungal Infections Cooperative Group and the National Institute of Allergy and Infectious Diseases Mycoses Study Group (EORTC/MSG) Consensus Group definitions [20]. The majority of infections (63\%) were proven via histopathologic, cytopathologic, or direct microscopic examination.

A total of 56 patients received AFMT as treatment for IA $(45.2 \%) ; 10$ patients received AFMT as treatment for IM (8.1\%); 5 patients received AFMT as treatment for both IA and IM (4.0\%); and 1 patient received AFMT as treatment for other IFI $(0.8 \%)$. A total of 17 patients received AFMT as prophylaxis for IA (13.7\%); 8 patients received AFMT as prophylaxis for IM (6.5\%); 10 patients received AFMT as prophylaxis for both IA and IM (8.1\%); and 8 patients received AFMT as prophylaxis for other IFI $(6.5 \%)$.

\section{Duration and Dosage of First-line AFMT}

Median duration of first-line AFMT was 14 days (range 1-603 days) for patients initiating AFMT as prophylaxis, and 19 days (range 3-351 days) for patients initiating AFMT as treatment (mean $[ \pm \mathrm{SD}] 60.0[ \pm 118.0]$ vs $40.5[ \pm 58.8] ; P=0.16)$ (Fig. 1). 
Table 1 Patient characteristics at hospitalization

\begin{tabular}{|c|c|c|c|}
\hline Characteristic & $\begin{array}{l}\text { Prophylaxis } \\
n=43\end{array}$ & $\begin{array}{l}\text { Treatment } \\
n=81\end{array}$ & $P$ value $^{\text {a }}$ \\
\hline \multicolumn{4}{|l|}{ Sex, $n(\%)$} \\
\hline Male & $27(62.8)$ & $52(64.2)$ & $>0.999$ \\
\hline Female & $16(37.2)$ & $29(35.8)$ & \\
\hline \multicolumn{4}{|l|}{ Age, years } \\
\hline Mean (SD) & $53.4(14.6)$ & $51.3(16.0)$ & 0.365 \\
\hline Median & 54.2 & 51.6 & \\
\hline Min-max & $23-77$ & $21-86$ & \\
\hline \multicolumn{4}{|l|}{ Race, $n(\%)$} \\
\hline White & $28(65.1)$ & $47(58.0)$ & 0.782 \\
\hline Black or African American & $10(23.3)$ & $15(18.5)$ & \\
\hline Asian & $5(11.6)$ & $15(18.5)$ & \\
\hline American Indian or Alaska Native & $0(0.0)$ & $1(1.2)$ & \\
\hline Other & $0(0.0)$ & $1(1.2)$ & \\
\hline No answer & $0(0.0)$ & $2(2.5)$ & \\
\hline \multicolumn{4}{|l|}{ BMI, $\mathrm{kg} / \mathrm{m}^{2}$} \\
\hline Mean (SD) & $26.6(3.3)$ & $25.9(3.6)$ & 0.201 \\
\hline Median & 25.8 & 25.5 & \\
\hline Min-max & $21.3-35.4$ & $21.0-40.4$ & \\
\hline \multicolumn{4}{|c|}{$\begin{array}{l}\text { Location of fungal infection manifestation } \\
\text { (occurring in }>10 \% \text { total population), } n(\%)^{\mathrm{b}}\end{array}$} \\
\hline Lung & $15(34.9)$ & $51(63.0)$ & 0.004 \\
\hline Blood & $7(16.3)$ & $34(42.0)$ & 0.005 \\
\hline Sinus & $9(20.9)$ & $18(22.2)$ & $>0.999$ \\
\hline Skin & $5(11.6)$ & $9(11.1)$ & $>0.999$ \\
\hline \multicolumn{4}{|c|}{$\begin{array}{l}\text { Primary underlying condition } \\
\text { (occurring in }>10 \% \text { total population), } n \text { (\%) }\end{array}$} \\
\hline Acute myeloid leukemia & $8(18.6)$ & $12(14.8)$ & 0.614 \\
\hline Hematopoietic stem cell transplantation & $8(18.6)$ & $10(12.3)$ & 0.424 \\
\hline Diabetes or uncontrolled hyperglycemia & $6(14.0)$ & $10(12.3)$ & 0.785 \\
\hline Solid-organ transplant & $5(11.6)$ & $10(12.3)$ & $>0.999$ \\
\hline
\end{tabular}


Table 1 continued

\begin{tabular}{|c|c|c|c|}
\hline Characteristic & $\begin{array}{l}\text { Prophylaxis } \\
n=43\end{array}$ & $\begin{array}{l}\text { Treatment } \\
n=81\end{array}$ & $P$ value $^{\text {a }}$ \\
\hline \multicolumn{4}{|c|}{ Risk factors (occurring in $>10 \%$ total population), $n(\%)^{\mathrm{b}}$} \\
\hline Prolonged neutropenia & $11(25.6)$ & $32(39.5)$ & 0.165 \\
\hline Fungal or bacterial infection & $9(20.9)$ & $26(32.1)$ & 0.214 \\
\hline Allogeneic stem cell transplantation & $8(18.6)$ & $12(14.8)$ & 0.614 \\
\hline Prolonged or high-dose use of corticosteroids & $5(11.6)$ & $12(14.8)$ & 0.786 \\
\hline Other form of infection & $7(16.3)$ & $8(9.9)$ & 0.386 \\
\hline No risk factors reported & $5(11.6)$ & $10(12.3)$ & $>0.999$ \\
\hline \multicolumn{4}{|c|}{ Comorbid conditions (occurring in $>10 \%$ total population), $n(\%)^{\mathrm{b}}$} \\
\hline Hematological malignancy & $19(44.2)$ & $27(33.3)$ & 0.248 \\
\hline Diabetes $^{c}$ & $7(16.3)$ & $18(22.2)$ & 0.489 \\
\hline Mild hepatic disease ${ }^{\mathrm{d}}$ & $3(7.0)$ & $16(19.8)$ & 0.070 \\
\hline Moderate pulmonary disease ${ }^{\mathrm{e}}$ & $5(11.6)$ & $14(17.3)$ & 0.448 \\
\hline Moderate to severe renal impairment ${ }^{\mathrm{f}}$ & $5(11.6)$ & $10(12.3)$ & $>0.999$ \\
\hline \multicolumn{4}{|l|}{ QTc prolongation, $n(\%)$} \\
\hline No & $38(88.4)$ & $72(88.9)$ & $>0.999$ \\
\hline Unknown & $5(11.6)$ & $9(11.1)$ & \\
\hline \multicolumn{4}{|l|}{ Prior antifungal therapy at any time, $n(\%)$} \\
\hline Yes & $8(18.6)$ & $14(17.3)$ & 0.853 \\
\hline No & $29(67.4)$ & $52(64.2)$ & \\
\hline Unknown & $6(14.0)$ & $15(18.5)$ & \\
\hline \multicolumn{4}{|l|}{ Antifungal agent received, $n(\%)$} \\
\hline Amphotericin B & $7(16.3)$ & $24(29.6)$ & 0.129 \\
\hline Isavuconazole & $9(20.9)$ & $22(27.2)$ & 0.518 \\
\hline Voriconazole & $11(25.6)$ & $20(24.7)$ & $>0.999$ \\
\hline Posaconazole & $16(37.2)$ & $15(18.5)$ & 0.030 \\
\hline
\end{tabular}

$B M I$ body mass index, max maximum, min minimum, $S D$ standard deviation

a Differences were assessed using the Kruskal-Wallis test for continuous variables and Fisher's exact test for categorical variables

b Multiple responses allowed

c Requiring insulin or oral hypoglycemics

d Chronic hepatitis, bilirubin $>$ upper limit of normal (ULN) to $1.5 \times$ ULN, or aspartate aminotransferase/alanine aminotransferase $>$ ULN to $2.5 \times$ ULN

e The diffusing capacity for carbon monoxide (DLCO) and/or forced expiratory volume in $1 \mathrm{~s}\left(\mathrm{FEV}_{1}\right)$ 66-80\% or dyspnea on slight activity

${ }^{\mathrm{f}}$ Creatinine clearance $<50 \mathrm{~mL} / \mathrm{min}$ 


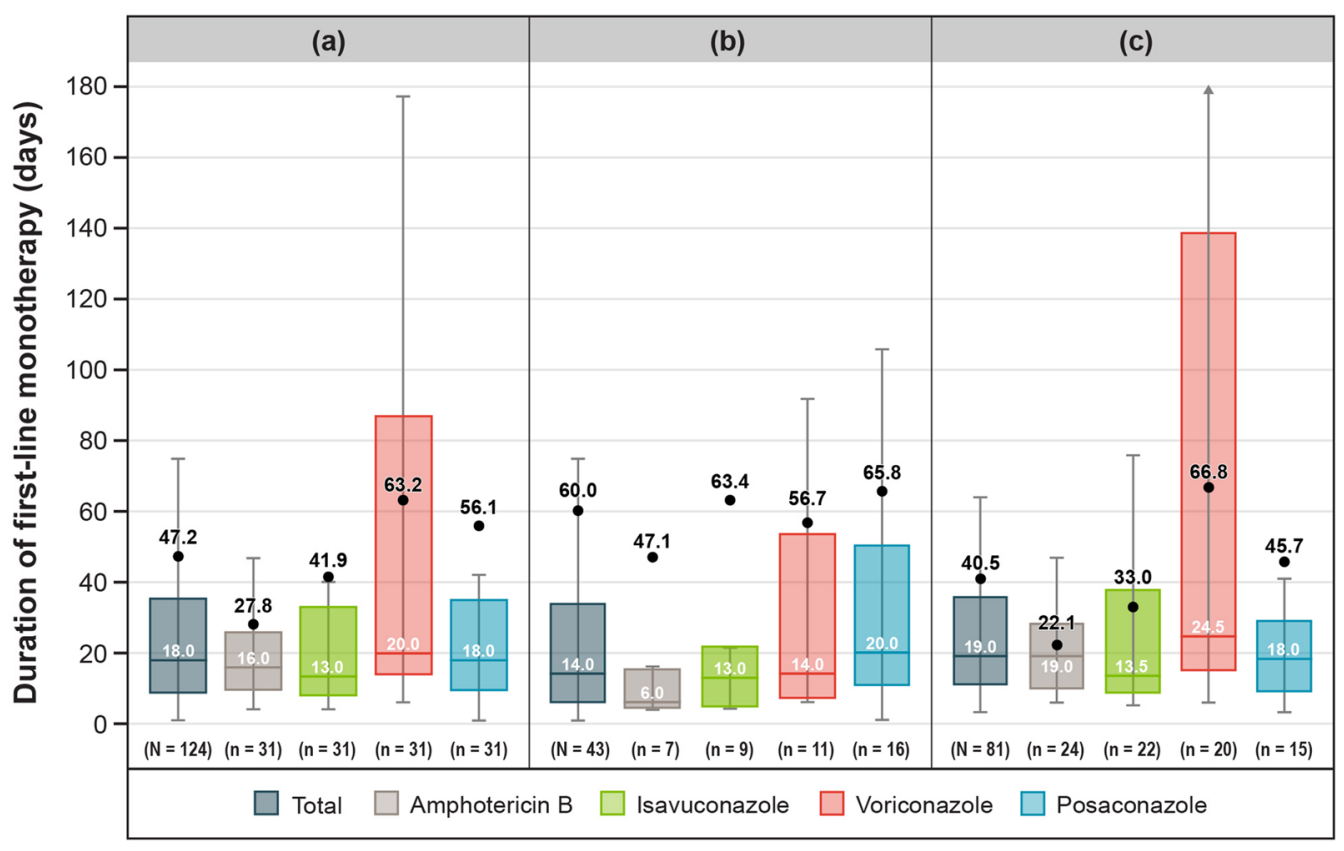

Fig. 1 Duration of antifungal monotherapy (AFMT). a Overall duration of first-line AFMT, total study population. b Duration of prophylaxis with first-line AFMT, prophylaxis group. $\mathbf{c}$ Duration of treatment with first-line AFMT, treatment group. Whiskers represent the minimum and maximum values excluding outliers; horizontal line represents the median; the upper and lower portions of the box represent the upper and lower quartiles; the circle represents the mean. Median daily

In the prophylaxis group, 7 patients received first-line amphotericin B for a median duration of 6 days (range 4-280 days) (Fig. 1b). The median daily dosage was $2.4 \mathrm{mg} / \mathrm{kg}$ (range 1.0-7.4 mg/kg) (recommended dose: 3-6 mg/ $\mathrm{kg}$ /day liquid formulation [18]). Nine patients received isavuconazole as prophylaxis for a median duration of 13 days (range 4-291 days) at a median daily dose of $372 \mathrm{mg}$ (range 20-1116 mg) (recommended dose: $372 \mathrm{mg} /$ day, after a 2-day loading regimen [18]). Eleven patients received voriconazole as prophylaxis for a median duration of 14 days (range 6-351 days) at a median daily dose of $240 \mathrm{mg}$ (range 5-800 mg) (recommended dose: $400 \mathrm{mg}$ /day [18]). Sixteen patients received posaconazole as prophylaxis for a median duration of 20 days (range 1-603 days) at a median daily dose of $300 \mathrm{mg}$ (range $15-600 \mathrm{mg}$ ) (recommended dose: $300 \mathrm{mg} /$ day [18]). dosage for prophylaxis: amphotericin $\mathrm{B}, 2.4 \mathrm{mg} / \mathrm{kg}$ (range $1.0-7.4 \mathrm{mg} / \mathrm{kg}$ ); isavuconazole, $\quad 372 \mathrm{mg}$ (range 20-1116 mg); voriconazole, $240 \mathrm{mg}$ (range 5-800 mg); posaconazole, $300 \mathrm{mg}$ (range $15-600 \mathrm{mg}$ ). Median daily dosage for treatment: amphotericin $\mathrm{B}, 2.3 \mathrm{mg} / \mathrm{kg}$ (range $0.2-6.7 \mathrm{mg} / \mathrm{kg}$ ); isavuconazole, $\quad 372 \mathrm{mg}$ (range $10-1116 \mathrm{mg}$ ); voriconazole, $\quad 400 \mathrm{mg} \quad$ (range $100-650 \mathrm{mg}$ ); posaconazole, $300 \mathrm{mg}$ (range $25-400 \mathrm{mg}$ )

In the treatment group, 24 patients received first-line amphotericin B for a median duration of 19 days (range 6-64 days) (Fig. 1c). The median daily dosage was $2.3 \mathrm{mg} / \mathrm{kg}$ (range $0.2-6.7 \mathrm{mg} / \mathrm{kg}$ ). Twenty-two patients received isavuconazole as treatment for a median duration of 14 days (range 5-165 days) at a median daily dose of $372 \mathrm{mg}$ (range 10-1116 mg). Twenty patients received voriconazole as treatment for a median duration of 25 days (range 6-217 days) at a median daily dose of $400 \mathrm{mg}$ (range 100-650 mg). Fifteen patients received posaconazole as treatment for a median duration of 18 days (range 3-351 days) at a median daily dose of $300 \mathrm{mg}$ (range $25-400 \mathrm{mg}$ ).

\section{Changes in Treatment or Prophylaxis Following Initiation}

Seven treatment changes were documented (Table 2). Two dose reductions were observed: 
one for a patient receiving amphotericin B due to nephrotoxicity and one for a patient receiving voriconazole for undocumented reasons. One dose increase due to lack of efficacy occurred in a patient receiving posaconazole. One treatment switch from isavuconazole to voriconazole occurred due to elevated liver enzymes. Treatment was discontinued in 3 patients due to lack of efficacy (amphotericin B) or for undocumented reasons (isavuconazole and voriconazole).

\section{Healthcare Resource Use}

\section{Duration of Initial Hospitalization}

Of the 43 records extracted related to prophylaxis, physicians provided complete hospitalization date information for 24 patients (56\%); of the 81 records related to treatment, physicians provided complete hospitalization date information for 65 patients (80\%) (Fig. 2a). Median duration of hospitalization was 29 days (range 4-259 days) in the prophylaxis group versus 31 days (range 6-980 days) in the treatment group (mean $[ \pm \mathrm{SD}]=37.8[ \pm 50.7]$ vs $84.6[ \pm 188.1] ; P=0.09)$. Patients initiated AFMT shortly after hospitalization. Median duration of hospitalization following AFMT initiation was 25 days (range 4-259 days) in the prophylaxis group versus 28 days (range 5-968 days) in the treatment group (mean $[ \pm \mathrm{SD}]=32.9 \quad[ \pm 51.2] \quad$ vs $79.1 \quad[ \pm 187.1]$; $P=0.07)$.

Among patients receiving prophylaxis, those initiating therapy with posaconazole or voriconazole were hospitalized for a median duration of 30 days (range 5-259 days) or 28 days (range 11-29 days), respectively, compared with 20 days (range 4-41 days) for amphotericin $\mathrm{B}$ and 10 days (range 4-35 days) for isavuconazole (Fig. 2b).

Among patients receiving treatment, those initiating therapy with voriconazole were hospitalized for a median of 42 days (range 6-135 days), compared with 32 days (range 7-147 days) for amphotericin B, 31 days (range 8-980 days) for posaconazole and 21 days (range 8-189 days) for isavuconazole (Fig. 2c).

Table 2 Changes in treatment or prophylaxis following initiation

\begin{tabular}{|c|c|c|c|c|}
\hline & $\begin{array}{l}\text { Amphotericin B } \\
n=31 \\
n(\%)\end{array}$ & $\begin{array}{l}\text { Isavuconazole } \\
n=31 \\
n(\%)\end{array}$ & $\begin{array}{l}\text { Voriconazole } \\
n=31 \\
n(\%)\end{array}$ & $\begin{array}{l}\text { Posaconazole } \\
n=31 \\
n(\%)\end{array}$ \\
\hline \multirow[t]{2}{*}{ Dose reduction } & $1(3.2)$ & $0(0.0)$ & $1(3.2)$ & $0(0.0)$ \\
\hline & $\begin{array}{l}\text { Due to } \\
\text { nephrotoxicity }\end{array}$ & & $\begin{array}{l}\text { Rationale } \\
\text { undocumented }\end{array}$ & \\
\hline \multirow[t]{2}{*}{ Dose increase } & $0(0.0)$ & $0(0.0)$ & $0(0.0)$ & $1(3.2)$ \\
\hline & & & & $\begin{array}{l}\text { Due to lack of } \\
\text { efficacy }\end{array}$ \\
\hline \multirow[t]{2}{*}{ Treatment switch } & $0(0.0)$ & $1(3.2)$ & $0(0.0)$ & $0(0.0)$ \\
\hline & & $\begin{array}{l}\text { Due to elevated liver } \\
\text { enzymes }\end{array}$ & & \\
\hline \multirow[t]{2}{*}{ Discontinuation } & $1(3.2)$ & $1(3.2)$ & $1(3.2)$ & $0(0.0)$ \\
\hline & $\begin{array}{l}\text { Due to lack of } \\
\text { efficacy }\end{array}$ & Rationale undocumented & $\begin{array}{l}\text { Rationale } \\
\text { undocumented }\end{array}$ & \\
\hline None & $25(80.6)$ & $26(83.9)$ & $28(90.3)$ & $29(93.5)$ \\
\hline Unknown & $4(12.9)$ & $3(9.7)$ & $1(3.2)$ & $1(3.2)$ \\
\hline
\end{tabular}




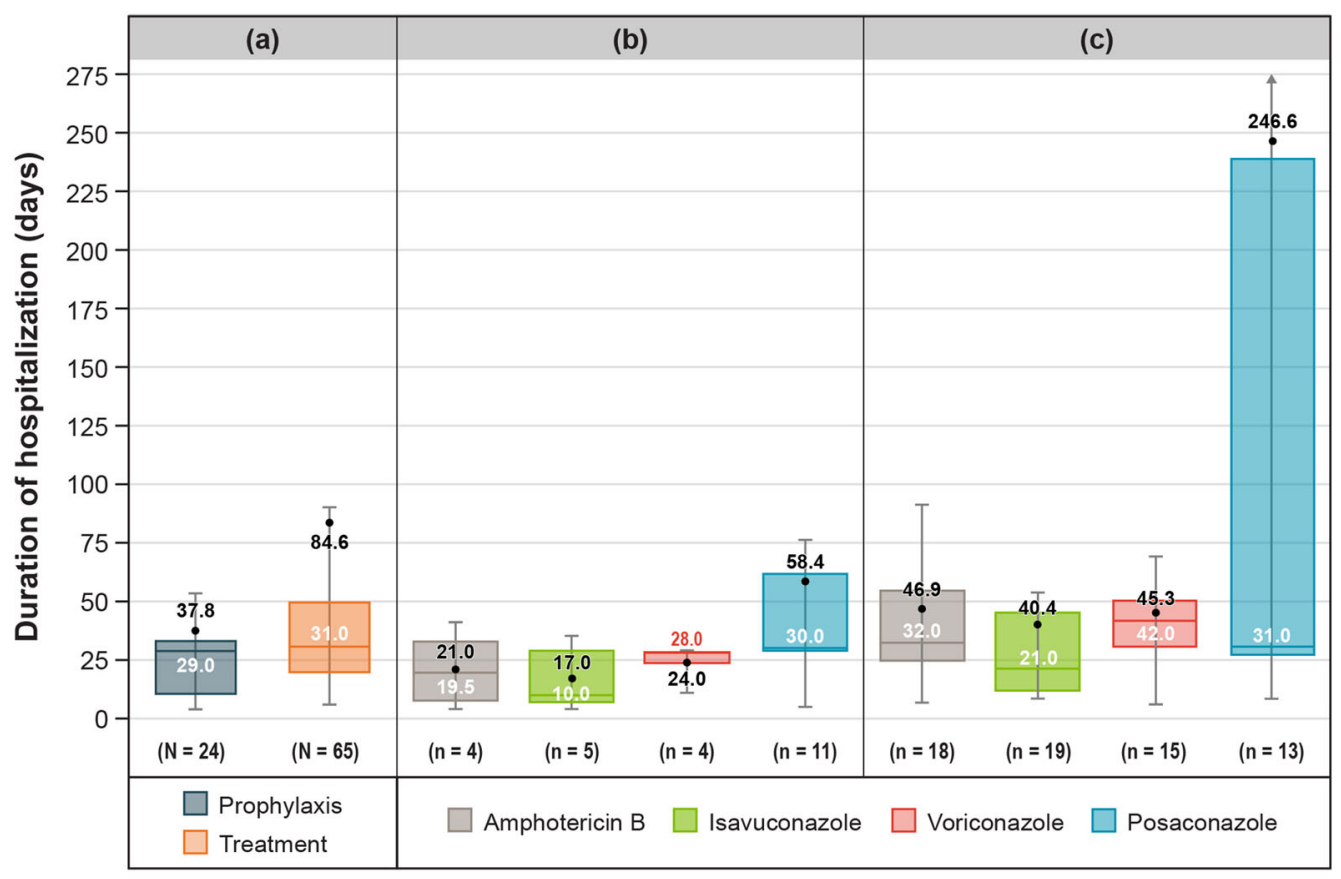

Fig. 2 Duration of initial hospitalization. a Overall duration of initial hospitalization, total study population. b Duration of initial hospitalization for patients receiving first-line AFMT as prophylaxis. c Duration of initial hospitalization for patients receiving first-line AFMT as treatment. Whiskers represent the minimum and maximum values excluding outliers; horizontal line represents

\section{Emergent Care During the Initial Hospitalization}

Mechanical ventilation was required by $5 \%$ of patients on prophylaxis and $31 \%$ of patients on treatment $(P=0.001)$. Mechanical ventilation use in patients receiving treatment was most common among patients receiving voriconazole $(45 \%$ vs $40 \%$ for posaconazole, $32 \%$ for isavuconazole, and 13\% for amphotericin B). Admission to the ICU occurred in $14 \%$ of patients on prophylaxis and $52 \%$ of patients on treatment $(P<0.001)$. ICU admission rates for patients receiving treatment were similar regardless of antifungal agent used (55\% for isavuconazole, $55 \%$ for voriconazole, $53 \%$ for posaconazole, and $46 \%$ for amphotericin B).

\section{Hospital Readmission Following Discharge}

Of the 106 patients who did not die during the initial hospitalization, 98 (92\%) had been the median; the upper and lower portions of the box represent the upper and lower quartiles; the circle represents the mean. Of the 124 patient records extracted, physicians provided complete and usable hospitalization date information for 89 patients. AFMT, antifungal monotherapy

discharged by the time of data retrieval. Physicians were aware of the readmission status of 63 of these discharged patients. One patient was readmitted within 30 days of discharge due to continued antifungal infection; this patient received isavuconazole as treatment during their initial hospitalization.

\section{Survival}

At the time of data retrieval, 31 patients (25\%) were known to have died (5 prophylaxis; 26 treatment). Overall survival rates were $88 \%$ (36/ $41)$ in the prophylaxis group and $66 \%(50 / 76)$ in the treatment group. Of the 31 deaths, 16 were reported to be caused by or related to IA or IM. Among patients receiving AFMT as prophylaxis, those who died were receiving either amphotericin B ( 2 patients), isavuconazole (1 patient), or voriconazole ( 2 patients). Among patients 
(a)

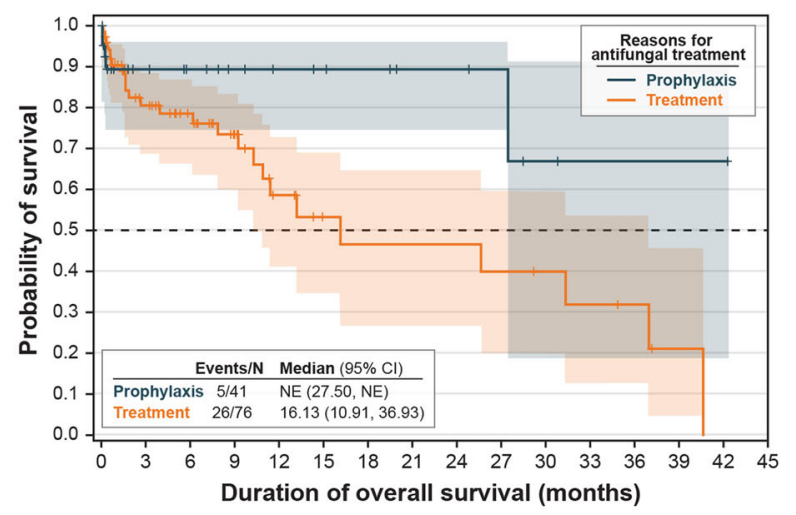

(b)

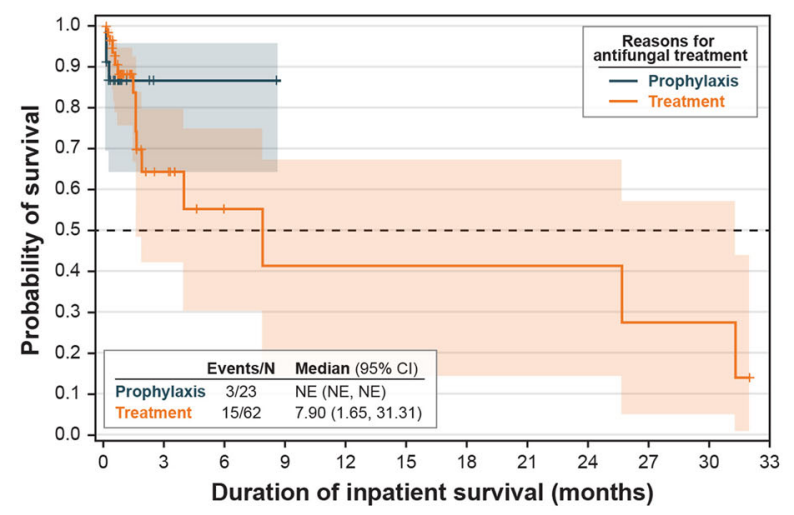

Fig. 3 Survival. a Overall survival by reason for AFMT initiation. b Inpatient survival by reason for AFMT initiation. Tick marks represent censoring; shaded areas represent the $95 \%$ confidence intervals. CI, confidence interval; NE, not established

receiving AFMT as treatment, deaths occurred more commonly among patients receiving voriconazole or posaconazole (9 and 8 patients, respectively) than among patients receiving isavuconazole or amphotericin B (6 and 3 patients, respectively). For patients with a known vital status and complete date information $(n=117)$, the median overall survival duration was not estimable for patients receiving prophylaxis and was 16.1 months (95\% confidence interval $[\mathrm{CI}], 10.9,36.9)$ for patients receiving treatment (Fig. 3a).

Eighteen of the 124 patients included in the study (15\%) were known to have died during hospitalization (3 prophylaxis; 15 treatment). At the time of data retrieval, inpatient survival rates were $87 \%(20 / 23)$ in the prophylaxis group and $76 \%(47 / 62)$ in the treatment group. For patients with a known vital status and complete date information $(n=85)$, the median duration of inpatient survival was not estimable for patients receiving prophylaxis and was 7.9 months (95\% CI, 1.7, 31.3) for patients receiving AFMT as treatment (Fig. 3b).

\section{DISCUSSION}

This retrospective cohort study has described the characteristics of adult inpatients with, or at risk of, IA or IM who initiated AFMT with amphotericin $\mathrm{B}$, isavuconazole, voriconazole, or posaconazole during their hospital stay. It is one of few studies to provide real-world data on the profile of such patients $[14,16,17,21]$ and is the first to document clinical practice and outcomes in a diverse population since the US introduction of isavuconazole. Our findings demonstrated that: IA/IM were associated with lengthy hospital stays commonly requiring ICU admission; prophylactic and therapeutic dosages and duration of AFMT generally followed published clinical guidelines [18]; and hospital readmission and switching between agents occurred rarely.

The demographic and clinical profiles of the study population were similar to those reported previously $[22,23]$. Most of the population were white males who were overweight, with fungal infections most commonly manifesting in the lungs, blood, and sinuses, and the coexistence of many comorbidities [24, 25]. The high level of heterogeneity in underlying diseases known to be associated with IA and IM was evident, with no one condition accounting for more than $20 \%$ of the sample.

The 2016 Infectious Diseases Society of America guidelines for the management of IA recommend triazoles as the preferred agents for treatment and prevention [18]. In our sample, amphotericin B, isavuconazole, and voriconazole were most commonly associated with treatment, while posaconazole was most commonly associated with prophylaxis.

Real-world data on the duration of antifungal prophylaxis and treatment in the inpatient 
setting are lacking. When used as treatment, the guidelines recommend that therapy be continued for a minimum of 6-12 weeks. In our study, therapy for treatment was received, on average, for just under 6 weeks. However, the data were skewed, with only 12 of the 81 patients receiving treatment for longer than 7.5 weeks, and thus the median treatment duration was just under 3 weeks. Most of the study population had been discharged by the start of our data retrieval period, with only one patient reported to have been readmitted to hospital within 30 days of discharge. Deaths related to IA and IM were uncommon.

Given the lack of real-world evidence about the duration of antifungal prophylaxis and treatment in the inpatient setting, further research is warranted to examine the extent to which the durations and outcomes identified are representative of common practice. Such information may be beneficial for the further development of treatment guidelines in the USA.

Our study revealed wide variation in AFMT dosages, particularly for isavuconazole (median dosage [range]: prophylaxis, 372 [20-1116] $\mathrm{mg} /$ day; treatment, 372 [10-1116] mg/day). This is interesting in light of a recently reported real-world case of a 23-year-old male with severe aplastic anemia in whom IA was successfully controlled through use of low-dose isavuconazole [26]. Blood concentrations of isavuconazole remained high after reducing the dose from $200 \mathrm{mg} /$ day to $100 \mathrm{mg} /$ day in response to increased liver function tests. This high concentration may be related to the patient taking cyclosporine, which has been shown to increase peak concentration of isavuconazole by $30 \%$ via inhibition of CYP3A4 [27].

Treatment switching in the inpatient setting has been studied in follow-on clinical trial settings [28] and in retrospective observational settings in which eligibility criteria involved patients who switched treatment [15], but little is known about the occurrence of and reasons for real-world treatment switching. Our results indicate that switching agents is rare among hospitalized patients in the USA with, or at risk of, IA or IM. Current treatment guidelines recommend salvage therapy with a different class of antifungal, tapering or reversal of underlying immunosuppression, and surgical removal of necrotic lesions [18]. Our study did not assess treatment of the underlying condition, so the extent to which non-antifungal treatment was tapered or reversed is unknown.

The overall mortality rate we identified in the treatment group $(32 \%$ [26/81] at the time of data retrieval) was similar to previously reported data from real-world settings [29-31]). However, our study reported a low in-hospital mortality rate $(19 \%[15 / 81])$ in the treatment group compared with previously reported mortality rates for ICU patients with IA (46-80\%) [25]. In our sample, $52 \%$ of the treatment group were admitted to the ICU, suggesting that the group as a whole were less sick than the patients in these previous reports. The low in-hospital mortality rate in the treatment group may also be explained in part by the fact that previous studies predate the availability of improved diagnostics, treatment guidelines, and the newer antifungal agents studied here. The diversity of our population in terms of underlying disease and the respective small sample sizes may also have contributed to the low inpatient mortality rate observed. In addition, the estimated survival results we have presented should be interpreted with caution because most of the censoring occurred in the first 12 months [32]. Moreover, no adjustments were made, and patients who were prescribed isavuconazole had a shorter opportunity for follow up compared with patients who were prescribed the other antifungal agents (i.e., the earliest available entry date for this study was 2013, but isavuconazole was not approved in the USA until 2015).

Retrospective medical record review studies are subject to limitations. The patients selected for study inclusion represented a convenience sample and therefore the findings may have limited generalizability. Data were limited to information available in the records, and information on services received outside the physician's care setting not recorded in the record were unavailable. Additionally, some data were missing, particularly date information. Although data checks were in place to assess the internal consistency of the entered 
data, an independent reviewer did not validate the responses; reliability was therefore not assessed. Further, the study did not assess the severity of patients' underlying conditions. It is plausible that some of the observed differences between the antifungal agents may reflect condition severity rather than prophylactic or therapeutic efficacy. In addition, this study involved small numbers of patients per antifungal agent, and the groups were noncontemporaneous; other host- and infectionrelated issues may therefore have influenced resource utilization and survival outcomes.

This study aimed to document patient profiles and treatment patterns associated with the use of each AFMT, and the associated healthcare resource utilization and survival outcomes. To allow the patterns and outcomes for each therapy to be examined, considering the rarity of the condition, we imposed selection criteria to ensure an equal number of patients received each AFMT. However, this approach does not reflect real-world use of AFMT, and our findings do not reflect the frequency with which each AFMT is used in the inpatient setting. Moreover, participating physicians must have prescribed each AFMT: physicians who prescribe a particular AFMT only were not included. It is plausible that different hospitals across the USA have different prescribing practices, for example, due to AFMT familiarity and access. Therefore, the study results cannot be used to inform understanding of the distribution of use of each AFMT across the USA.

Our study has focused on patients with, or at risk of, IA or IM who received AFMT. However, combination therapy is now used increasingly to treat IFIs in attempts to improve outcomes, particularly for more severe cases. A randomized controlled trial in patients with hematological malignancies or hematopoietic cell transplantation has reported that treatment of IA with voriconazole plus anidulafungin was associated with a statistically nonsignificant but clinically meaningful survival benefit compared with voriconazole alone [33]. A recent small retrospective chart review of medical records has reported a significantly lower mortality rate in patients with non-Aspergillus IFIs, including mucormycosis, treated with combination antifungal therapy compared with AFMT [34]. If, indeed, severe IA/IM cases tend to receive combination antifungal therapy, the methods employed in our study may have introduced a bias by selecting less severe cases receiving AFMT and patients receiving AFMT as prophylaxis. This study is the first to document realworld, inpatient, prophylactic and therapeutic use of antifungal agents for IFIs and the associated outcomes since isavuconazole was approved in the USA. The findings provide valuable evidence of contemporary practices that warrant further investigation using alternative data sources such as patient registries. Research already underway aims to build upon the current findings by extending study to the outpatient setting and by replicating our approach in a large registry of patients treated with systemic mold-active triazoles in the USA (ClinicalTrials.gov identifier: NCT03066011).

\section{CONCLUSIONS}

This retrospective medical record review has described the characteristics of a sample of hospitalized patients with, or at risk of, IA or IM in the USA. In addition, the real-world use of mold-active triazoles for prevention and treatment of IA/IM, as well as associated outcomes, were documented. IA and IM most commonly manifested in the lungs, blood, and sinuses, and were associated with lengthy hospital stays that frequently required ICU admission. Real-world prophylaxis and treatment generally followed published clinical guidelines. Subsequent hospital readmission and switching between agents were rare. These findings highlight the importance of IFI monitoring during hospitalization and the use of appropriate prophylaxis and treatment.

\section{ACKNOWLEDGEMENTS}

The authors wish to acknowledge Samuel Wilson, Ph.D., and Janet Kim, Ph.D., at Astellas Pharma Global Development, Inc., for their helpful reviews of the study protocol and statistical analysis plan. 
Funding. This work and the rapid service fees were supported by Astellas Pharma Global Development, Inc., Northbrook, IL, USA. All authors had full access to all the data in this study and take complete responsibility for the integrity of the data and accuracy of the data analysis.

Medical Writing and Editorial Assistance. Editorial assistance in the preparation of this article was provided by Fiona Weston and Sarah Whitfield, PhD, for Cello Health MedErgy. Support for this assistance was funded by Astellas Pharma Global Development, Inc., Northbrook, IL, USA.

Authorship. All named authors meet the International Committee of Medical Journal Editors (ICMJE) criteria for authorship for this article, take responsibility for the integrity of the work as a whole, and have given their approval for this version to be published.

Disclosures. Katherine Stull is an employee of RTI Health Solutions, which received consultancy fees for study execution and medical writing. Elizabeth Esterberg is an employee of RTI Health Solutions, which received consultancy fees for study execution and medical writing. Mayank Ajmera is an employee of RTI Health Solutions, which received consultancy fees for study execution and medical writing. Sean Candrilli is an employee of RTI Health Solutions, which received consultancy fees for study execution and medical writing. Therese M. Kitt is an employee of Astellas Pharma Global Development, Inc. James R. Spalding is an employee of Astellas Pharma Global Development, Inc. Vanessa Perez Patel was an employee of Astellas Pharma Global Development, Inc., at the time of the analysis. Her current affiliation is Covance Market Access, 9801 Washingtonian Blvd., 9th Floor, Gaithersburg, MD 20878, USA.

Compliance with Ethics Guidelines. All procedures performed in studies involving human participants were in accordance with the ethical standards of RTI International's institutional review board (Research Triangle Park, North Carolina) and with the 1964
Helsinki declaration and its later amendments or comparable ethical standards. Informed consent was not sought due to the nature of the study: all retrieved data were anonymous and were those collected as part of routine diagnosis and treatment. There was no effect of the review on patient care. Physicians abstracting the data were treating physicians who had legitimate access to the medical records. RTI International's institutional review board determined that this study met all criteria for exemption from ethical considerations.

Data Availability. Access to anonymized individual participant level data will not be provided for this study as it meets one or more of the exceptions described on http://www. clinicalstudydatarequest.com under "Sponsor Specific Details for Astellas".

Open Access. This article is distributed under the terms of the Creative Commons Attribution-NonCommercial 4.0 International License (https://creativecommons.org/licenses/ by-nc/4.0/), which permits any noncommercial use, distribution, and reproduction in any medium, provided you give appropriate credit to the original author(s) and the source, provide a link to the Creative Commons license, and indicate if changes were made.

\section{REFERENCES}

1. Badiee P, Hashemizadeh Z. Opportunistic invasive fungal infections: diagnosis \& clinical management. Indian J Med Res. 2014;139(2):195-204.

2. Donnelley MA, Zhu ES, Thompson GR III. Isavuconazole in the treatment of invasive aspergillosis and mucormycosis infections. Infect Drug Resist. 2016;9:79-86.

3. Soubani AO, Chandrasekar PH. The clinical spectrum of pulmonary aspergillosis. Chest. 2002;121(6):1988-99.

4. Gavaldà J, Meije Y, Fortún J et al, ESCMID Study Group for Infections in Compromised Hosts. Invasive fungal infections in solid organ transplant recipients. Clin Microbiol Infect. 2014;20(Suppl 7):27-48. 
5. Kimura S. Invasive aspergillosis in hematological patients. Med Mycol J. 2016;57(2):J77-88.

6. Chamilos G, Luna M, Lewis RE, et al. Invasive fungal infections in patients with hematologic malignancies in a tertiary care cancer center: an autopsy study over a 15-year period (1989-2003). Haematologica. 2006;91(7):986-9.

7. Kontoyiannis DP, Yang H, Song J, et al. Prevalence, clinical and economic burden of mucormycosisrelated hospitalizations in the United States: a retrospective study. BMC Infect Dis. 2016;16(1):730.

8. Pagano L, Caira M, Candoni A, et al. Invasive aspergillosis in patients with acute myeloid leukemia: SEIFEM-2008 registry study. Haematologica. 2010;95(4):644-50.

9. Pappas PG, Alexander BD, Andes DR, et al. Invasive fungal infections among organ transplant recipients: results of the Transplant-Associated Infection Surveillance Network (TRANSNET). Clin Infect Dis. 2010;50(8):1101-11.

10. Vallabhaneni S, Benedict K, Derado G, Mody RK. Trends in hospitalizations related to invasive aspergillosis and mucormycosis in the United States, 2000-2013. Open Forum Infect Dis. 2017;4(1):ofw268.

11. Maertens JA, Raad II, Marr KA, et al. Isavuconazole versus voriconazole for primary treatment of invasive mould disease caused by Aspergillus and other filamentous fungi (SECURE): a phase 3, randomised-controlled, non-inferiority trial. Lancet. 2016;387(10020):760-9.

12. Herbrecht R, Denning DW, Patterson TF, et al. Voriconazole versus amphotericin B for primary therapy of invasive aspergillosis. N Engl J Med. 2002;347(6):408-15.

13. Walsh TJ, Raad I, Patterson TF, et al. Treatment of invasive aspergillosis with posaconazole in patients who are refractory to or intolerant of conventional therapy: an externally controlled trial. Clin Infect Dis. 2007;44(1):2-12.

14. Azie N, Neofytos D, Pfaller M, Meier-Kriesche H-U, Quan S-P, Horn D. The PATH (prospective antifungal therapy) Alliance ${ }^{\circledR}$ registry and invasive fungal infections: update 2012. Diagn Microbiol Infect Dis. 2012;73(4):293-300.

15. Heinz WJ, Egerer G, Lellek H, Boehme A, Greiner J. Posaconazole after previous antifungal therapy with voriconazole for therapy of invasive aspergillus disease, a retrospective analysis. Mycoses. 2013;56(3):304-10.

16. Chou LS, Lewis RE, Ippoliti C, Champlin RE, Kontoyiannis DP. Caspofungin as primary antifungal prophylaxis in stem cell transplant recipients. Pharmacotherapy. 2007;27(12):1644-50.

17. Kung H, Johnson MD, Drew RH, Saha-Chaudhuri P, Perfect JR. Clinical effectiveness of posaconazole versus fluconazole as antifungal prophylaxis in hematology-oncology patients: a retrospective cohort study. Cancer Med. 2014;3:667-73.

18. Patterson TF, Thompson GR III, Denning DW, et al. Practice guidelines for the diagnosis and management of aspergillosis: 2016 update by the Infectious Diseases Society of America. Clin Infect Dis. 2016;63(4):e1-60.

19. Matt $V$, Matthew $H$. The retrospective chart review: important methodological considerations. J Educ Eval Health Prof. 2013;10:12.

20. De Pauw B, Walsh TJ, Donnelly JP, et al. Revised definitions of invasive fungal disease from the European Organization for Research and Treatment of Cancer/Invasive Fungal Infections Cooperative Group and the National Institute of Allergy and Infectious Diseases Mycoses Study Group (EORTC/ MSG) Consensus Group. Clin Infect Dis. 2008;46(12):1813-21.

21. Wingard JR, Kubilis P, Lee L, et al. Clinical significance of nephrotoxicity in patients treated with amphotericin B for suspected or proven aspergillosis. Clin Infect Dis. 1999;29(6):1402-7.

22. Zilberberg MD, Shorr AF, Huang H, Chaudhari $\mathrm{P}$, Paly VF, Menzin J. Hospital days, hospitalization costs, and inpatient mortality among patients with mucormycosis: a retrospective analysis of US hospital discharge data. BMC Infect Dis. 2014;14(1):310.

23. Taccone FS, Van den Abeele A-M, Bulpa P, et al. Epidemiology of invasive aspergillosis in critically ill patients: clinical presentation, underlying conditions, and outcomes. Crit Care. 2015;19(1):7.

24. Lortholary O, Gangneux J, Sitbon K, et al., French Mycosis Study Group. Epidemiological trends in invasive aspergillosis in France: the SAIF network (2005-2007). Clin Microbiol Infect. 2011;17(12):1882-9.

25. Baddley JW, Ephens JM, Ji X, Gao X, Schlamm HT, Tarallo M. Aspergillosis in intensive care unit (ICU) patients: epidemiology and economic outcomes. BMC Infect Dis. 2013;23:13-29.

26. Hoenigl $M$, Prattes J, Neumeister P, Wölfler A, Krause R. Real-world challenges and unmet needs in the diagnosis and treatment of suspected invasive pulmonary aspergillosis in patients with haematological diseases: an illustrative case study. Mycoses. 2018;61(3):201-5. 
27. Groll AH, Desai A, Han D, et al. Pharmacokinetic assessment of drug-drug interactions of isavuconazole with the immunosuppressants cyclosporine, mycophenolic acid, prednisolone, sirolimus, and tacrolimus in healthy adults. Clin Pharmacol Drug Dev. 2017;6(1):76-85.

28. Patterson TF, Boucher HW, Herbrecht R, et al. Strategy of following voriconazole versus amphotericin B therapy with other licensed antifungal therapy for primary treatment of invasive aspergillosis: impact of other therapies on outcome. Clin Infect Dis. 2005;41(10):1448-52.

29. Jacobs F, Selleslag D, Aoun M, Sonet A, Gadisseur A. An observational efficacy and safety analysis of the treatment of acute invasive aspergillosis using voriconazole. Eur J Clin Microbiol Infect Dis. 2012;31(6):1173-9.

30. Kim A, Nicolau DP, Kuti JL. Hospital costs and outcomes among intravenous antifungal therapies for patients with invasive aspergillosis in the United States. Mycoses. 2011;54(5):e301-12.
31. Slobbe L, Polinder S, Doorduijn JK, et al. Outcome and medical costs of patients with invasive aspergillosis and acute myelogenous leukemia-myelodysplastic syndrome treated with intensive chemotherapy: an observational study. Clin Infect Dis. 2008;47(12):1507-12.

32. Rich JT, Neely JG, Paniello RC, Voelker CC, Nussenbaum B, Wang EW. A practical guide to understanding Kaplan-Meier curves. Otolaryngol Head Neck Surg. 2010;143(3):331-6.

33. Marr KA, Schlamm HT, Herbrecht R, et al. Combination antifungal therapy for invasive aspergillosis: a randomized trial. Ann Intern Med. 2015;162(2):81-9.

34. Jenks JD, Reed SL, Seidel D, et al. Rare mould infections caused by Mucorales, Lomentospora prolificans and Fusarium, in San Diego, CA: the role of antifungal combination therapy. Int J Antimicrob Agents. 2018;52(5):706-12. 